\title{
Relationship between pre-season anthropometric and fitness measures and indicators of playing performance in elite junior Australian Rules football
}

\& Warren B. Young*, Luke Pryor

9 School of Human Movement \& Sport Sciences, University of Ballarat, P.O. Box 663, Ballarat,

10 Victoria 3353, Australia

11 Received 6 December 2005; received in revised form 2 June 2006; accepted 4 June 2006

\footnotetext{
* Corresponding author. Tel.: +61 35327 9685; fax: +61 353279478.

E-mail address: w.young@ballarat.edu.au (W.B. Young).
} 


\section{Introduction}

Australian Rules football (ARF) is a fast moving field game involving players of varying body types. It requires a unique mix of physical, technical, mental and tactical skills. Elite juniors are drafted into the professional competition, the Australian Football League (AFL) each year and this selection process is partly influenced by test results from the National draft camp. This camp includes various forms of player assessment including physiological and anthropometric testing, with the results being made available to each AFL club.

Although physical fitness and anthropometric measures are believed to be related to playing performance, there is little data to describe this relationship. One way to describe the physical demands of AFL football is to analyse player movement patterns in competition. A recent comprehensive report indicated that typical play involved many short (less than 6s) high intensity efforts interspersed with walking and jogging movements. ${ }^{1}$

An alternative approach to evaluating the fitness requirements of football is to compare elite and sub-elite performers. Young et al. ${ }^{2}$ reported that the players from one AFL club selected to play at the start of the season were superior to nonselected players in pre-season levels of leg power, sprinting speed and performance in the Yo Yo intermittent recovery test. However the selected players were not significantly different in height and body mass, or in fitness measures such as lower and upper body strength or the $20 \mathrm{~m}$ multi-stage fitness test. These findings were restricted to one AFL club and may have limited application to elite junior footballers.

Some research on anthropometric and fitness measures has been conducted on junior ARF. Marchant and Austin ${ }^{3}$ compared senior AFL players with elite under-18 players on a variety of fitness tests, and reported no significant differences between the groups in height, body mass, sum of skinfolds, sprint times and vertical jump (VJ). However, the AFL players were superior in endurance, based on the $20 \mathrm{~m}$ multi-stage fitness test, as well as bench press strength.

Keogh ${ }^{4}$ compared selected and non-selected junior footballers aged 14-17. It was reported that there were no significant differences in pre-season measures of endurance or sit and reach, but the selected players were significantly taller, had superior bench press strength $(p<0.05)$ and tended to have better VJ to the non-selected group $(p<0.06)$. These studies involving junior footballers provide some insights into important physical qualities but were published in the 1990s, and game demands may have changed.

Pyne et al. $^{5}$ investigated the association between recent AFL draft camp test results and five measures of AFL career progression. While anthropometric variables had little relationship with career progression, there was a small but significant association with the $20 \mathrm{~m}$ sprint, jumping ability, agility and the $20 \mathrm{~m}$ multistage shuttle run.

While the above research has yielded some insights into the anthropometric and physical fitness measures associated with various levels of playing performance, there has been no research that has directly related the pre-season physical player profile to indicators of playing performance. Since there is no one accepted measure of on-field playing performance, we have used a range of individual player performance indicators from game statistics, and team standings on the ladder after eight games. The purpose of this research was to determine the relationship between various anthropometric and fitness measures obtained during the pre-season to indicators of playing performance during the early part of the season in elite junior ARF players. The results of this study should assist coaching staff to determine the relative importance of selected anthropometric and fitness parameters for performance and enhance the interpretation of test results from draft camps.

\section{Methods}

\section{Subjects}

Participants were 485 male players aged between 16 and 18 years and listed in the squads of the 12 Victorian clubs participating in the Transport Accident Commission (TAC) Cup under-18 football competition. The subjects were requested to participate in the test battery as part of their commitment to their club squad activities. Some players did not participate in all tests due to a form of injury at the time of testing.

\section{Testing}

The selected tests were modified from the AFL National draft camp and were prescribed by the administering body of the TAC Cup competition. All 12 Victorian clubs were tested in the pre-season between February 28 and March 23, 2005. The first game of the season was April 2-3, 2005. The venue for testing was chosen by each club but was always an indoor hall with a wooden floor surface. Each club performed their own warm-up which was 
supervised by the club's fitness coach. Although the warm-up protocols were not standardized, an advantage of this is that the players were familiar with the activities used.

\section{Anthropometric tests}

Standing height was determined with a stadiometer with a measurement resolution of $0.1 \mathrm{~cm}$. The player stood with the feet together without shoes, looked straight ahead and inhaled while the measurement was taken.

Body mass was determined with digital scales (Seca 770, Hamburg, Germany) that had a measurement resolution of $0.1 \mathrm{~kg}$. The player stood still on the scales wearing shorts, a t-shirt and socks, but no shoes.

Arm length was determined first by locating the acromion of the scapula by palpation. This was marked with a pen and the arm length was recorded as distance from this point to the middle fingertip with the arm extended and held parallel to the floor. The measurement was made with a plastic tape and recorded to the nearest $1 \mathrm{~cm}$.

Standing reach was assessed by standing the player underneath a Yardstick measurement device (Swift Performance Equipment, Lismore, Australia) with the feet together and flat on the floor. The player reached as high as possible with the preferred arm while the other arm remained by the side. The maximum reach height above the ground was recorded to a resolution of $1 \mathrm{~cm}$.

Hand span was measured for the right hand by placing the hand on a table so that the thumb lined up with the zero end of a steel ruler. The subject was asked to spread the hand as much as possible the distance from the thumb to the tip of the little finger was recoded to the nearest $0.1 \mathrm{~cm}$.

\section{Fitness tests}

A $5 \mathrm{~m}$ sprint time was assessed from a 20 sprint from a standing start. The front toe was placed on a start line and the player commenced the sprint when they were ready. The time was measured by a dual beam electronic timing system (Swift Performance Equipment, Lismore, Australia) with the start gate aligned with the start line and a gate placed at $5 \mathrm{~m}$ from the start. When the player ran through this gate, a $5 \mathrm{~m}$ time was recorded to a resolution of $0.01 \mathrm{~s}$. Players were required to commence their sprint from a stationary position; i.e. they were not permitted to use a rolling start.

A $20 \mathrm{~m}$ sprint time was assessed in the same way as for the $5 \mathrm{~m}$ time, but a finish gate placed at $20 \mathrm{~m}$ from the start recorded the total time. The fastest

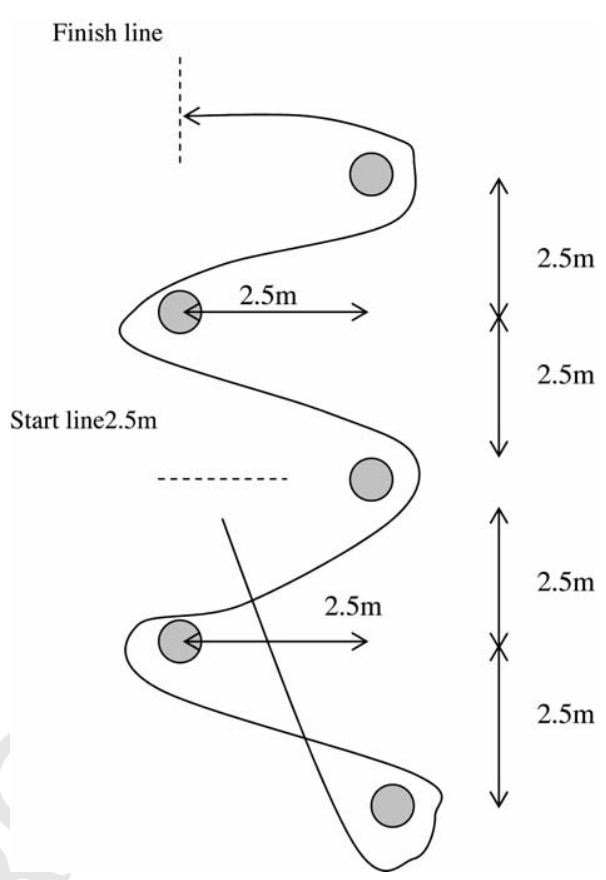

Figure 1 AFL agility test.

of three trials was recorded as the criterion score ${ }_{169}$ for both the 5 and $20 \mathrm{~m}$ times.

\section{AFL agility test}

This is a unique test consisting of a series of five planned changes of direction around poles (Fig. 1). The poles were $1.1 \mathrm{~m}$ high with a diameter of $12 \mathrm{~cm}$. The same timing system as that used for the sprints was used for this test with the faster of two trials used as the criterion score.

\section{A $20 \mathrm{~m}$ multi-stage shuttle run}

This test was conducted in accordance with the procedures outlined by Ellis et al. ${ }^{6}$ and the level and shuttle achieved was converted to a predicted $\dot{V}_{2 \text { max }}$ from the tables in Ramsbottom et al. ${ }^{7}$

\section{Standing vertical jump}

This was a jump from both legs performed with an arm swing and countermovement, but no preliminary steps were allowed. The player started in a fully extended position, dipped downward to a self-selected depth and then jumped as high as possible to displace the vanes on the Yardstick. The score was recorded as the difference between the standing reach and the highest point reached in the jump. Generally, three trials were allowed but if a player improved each trial, they were allowed further jumps until no further improvement occurred. 
Sit and reach (traditional)

The subject sat on the floor with the legs extended in front of the body and the trunk aligned vertically against a wall. The feet without shoes were held flat against the sit and reach box. The knees were kept fully extended and the player reached forward with the arms extended and one hand over the other. Although the shoulders were allowed to move forward, the back and head were required to remain in contact with the wall. The position of the fingertips was recorded as a baseline measure. The player then exhaled and reached as far forward as possible by flexing at the hips. The score recorded was the distance between the fingertips and the feet.

\section{Sit and reach (reach)}

Using the measurements from above, this score was expressed as the difference between the baseline value and reach position of the fingertips, to account for individual differences in baseline values.

The exact order of the tests was not identical for all clubs, although the anthropometric tests were always conducted first. This was generally followed by the sprint, agility and VJ in various orders. The variation in the order of tests was not considered a problem since adequate time for recovery between trials and tests was always allowed. Approximately $5 \mathrm{~s}$ rest between jumps and 2 min between sprints was provided to minimise fatigue. The multi-stage shuttle run was always conducted last. At the conclusion of all testing, players were recommended to perform their preferred cool-down.

\section{Game performance indicators}

Measurement of team or individual player performance is difficult in team sports such as ARF. Therefore, a number of player and team performance indicators were selected for analysis. These indicators were obtained from the first eight games and it was felt that this number of games would be a long enough time for the team standings (ladder) to become "settled" and also for player performance indicators to distinguish the better and worse performers. In addition, we chose to restrict the analysis to the early part of the season because pre-season fitness levels would be less likely to be related to performance during the latter part of the season when fitness levels are likely to be different. The eighth game was played within 3 months of the pre-season testing.

\section{Selected and non-selected players for game one} The "selected" players were the 18 players selected by each club to start on the field. Non- selected payers were required to be available for selection; i.e. not absent, ill or injured. This information was provided by a member of the coaching staff from each club. Generally, each club selected their better players and did not specifically use the pre-season fitness test results as a criterion for selection. Although the perceived playing ability of the available players was the major selection criterion, some clubs indicated that they also gave priority to the older players. This procedure of classifying players into groups on the basis of selection has previously been used in ARF, and has been shown to yield some significant differences in fitness measures. ${ }^{2}$

\section{Number of possessions}

A possession was considered to be either an effective kick or effective handball. An effective kick was defined as a long kick over $40 \mathrm{~m}$ to a $50 / 50$ contest or better or a short kick less than $40 \mathrm{~m}$ to an uncontested possession. Although the achievement of a higher number of possessions does not guarantee playing success, it was considered an indicator of the potential to be an effective player. An attempt was made to compare two groups on the basis of their average number of possessions. This was done to generate two groups for statistical comparison and as a result, the "high" possession group averaged more than 15 possessions per game (mean $=18.6 \pm 3.1$ ) and the "low" possession group averaged less than 15 possessions per game $($ mean $=11.0 \pm 2.5)$. This classification generally produced similar group sizes and were significantly different $(p<0.01)$ in average possessions/game.

\section{Number of marks}

Players were divided into two groups based on the average number of marks. If this number was greater than 3, the player was assigned to the "high" group, whereas less than 3 average marks per game resulted in assignment to the "low" group. This classification produced mean results of $4.4 \pm 1.1$ and $2.0 \pm .6$ for the high and low groups, respectively, and this difference was significant $(p<0.01)$.

\section{Number of hitouts}

A comparison was also made between groups on the average number of hitouts. The "high" group consisted of players who achieved more than six hitouts per game whereas the "low" group had to achieve one to five hitouts per game. Players who achieved less than one hitout per game on average over the first eight games were not used in the analysis because it was considered that these were 
players that do not normally participate in ruck contests. The high group averaged $14.0 \pm 1.8$ hitouts per game compared to $2.7 \pm 1.5$ for the low group $(p<0.01)$. The number of hitouts, as well as the number of possessions and marks was recorded in real time by club representatives at each game.

\section{Number of games where votes were awarded}

The match committee of each club awarded votes to the six players in their team considered to be the best players in each game. There may be many criteria for awarding votes and these are subjective in nature. Despite this, we believe it was reasonable to classify high, medium and low vote winners as an indicator of playing performance. The available data on votes was expressed as the number of games when votes were awarded. Players were assigned to the "high"' group if they achieved votes in four to eight games, the "medium" group if they were awarded votes in two to three games, and the "low" group if they were awarded votes in zero to one games. The number of games when votes were awarded rewards players who were consistently one of the top six performers compared to a measure of the total votes accumulated.

Effective possessions, number of marks and hitouts and the number of games where votes were awarded were recorded from game statistics by staff from each club, and tabulated by the competition organizing body (http://taccup. footballvic.com.au). Only players who played in all of the first eight games of the season were considered for the number of possessions, marks, hitouts and the number of games when votes were awarded.

\section{Top and bottom four teams}

While the above performance indicators relate to individual player performance, this measure was an attempt to compare groups based on team performance after eight games. To differentiate between the better and worse performing teams, the top and bottom four placed teams on the ladder were compared. After eight rounds, the total number of games won for the top four teams was 26 (mean per team $=6.5$ ), compared to only six games for the bottom four teams (mean per team $=1.5$ ). Only players who played in all of the first eight games of the season were used in this analysis.

\section{Statistics}

Classification into two or three groups was made on all performance indicators. To compare these groups on all anthropometric and fitness measures, a one-way analysis of variance (ANOVA) was per- formed. In the case of the three groups classified according to the awarding of votes, Tukey HSD multiple comparisons were performed to reveal where significant group differences if any, existed. Pearson correlations were also calculated to describe the inter-relationships among the variables. Statistical significance was set at $p<0.05$. Since statistical significance is influenced by sample size, we also calculated effect sizes $(E S)^{8}$ to view differences between groups. Effect sizes were interpreted as trivial $<0.2$, small $0.2-0.5$, medium $0.5-0.8$ and large $>0.8$ adapted from Cohen. ${ }^{8}$

\section{Results}

The comparison of anthropometric and fitness measures of selected and non-selected players is shown in Table 1. A comparison of statistically significant differences $(p<0.05)$ between the high and low possession groups is shown in Table 2 . The higher possession group was shorter and lighter and exhibited better sprint and endurance results. The only variable to significantly differentiate between the high and low groups with respect to the number of marks was body mass $(p=0.014, \mathrm{ES}=0.54)$, with the high group being $4.5 \mathrm{~kg}$ heavier $(80.8 \pm 9.4 \mathrm{~kg})$ than the low group $(76.3 \pm 7.2 \mathrm{~kg})$. The two hitout groups contained approximately 10 players each and the only variable that was significantly different $(p=0.004)$ between the groups and had a large ES (1.51) was height, with the higher hitout group being $5.7 \mathrm{~cm}$ taller $(195.0 \pm 4.1 \mathrm{~cm})$ than the lower group $(189.3 \pm 3.4 \mathrm{~cm})$. The results for the high, medium and low vote winners are shown in Table 3. The high and medium vote winners were significantly faster based on the 5 and $20 \mathrm{~m}$ sprint times than the low group. There were minimal differences in pre-season anthropometric and fitness measures between the top and bottom four teams (Table 4). However the better performing teams had significantly greater body mass, standing reach and inferior vertical jumping ability.

\section{Discussion}

This study is unique in that it focused on the relationship between pre-season anthropometric and fitness test measures and indicators of playing performance in Australian Rules football. Of major interest was whether more successful players and teams would be better in the pre-season scores. Several studies have reported significant differences between playing groups in certain fitness parameters when the groups are of vastly different 
Table 1 Comparison of selected and non-selected players for round one (mean \pm S.D.)

\begin{tabular}{lcccc}
\hline & Selected $(n=177-200)$ & Non-selected $(n=125-154)$ & $p$-Value & Effect size \\
\hline Anthropometric & & & & \\
$\quad$ Height $(\mathrm{cm})$ & $183.9 \pm 6.9$ & $182.9 \pm 6.2$ & 0.169 & 0.15 \\
Mass $(\mathrm{kg})$ & $79.8 \pm 8.3$ & $76.2 \pm 7.7$ & 0.001 & 0.44 \\
Hand span (cm) & $23.1 \pm 1.3$ & $22.6 \pm 1.3$ & 0.002 & 0.32 \\
Arm length (cm) & $80.1 \pm 4.8$ & $79.7 \pm 4.3$ & 0.445 & 0.09 \\
Standing reach (cm) & $238.5 \pm 10.0$ & $237.6 \pm 8.8$ & 0.388 & 0.10 \\
Fitness & & & & \\
VJ (cm) & $60.6 \pm 5.5$ & $58.1 \pm 6.0$ & 0.001 & 0.44 \\
$5 \mathrm{~m}$ time (s) & $1.12 \pm .05$ & $1.14 \pm .05$ & 0.018 & 0.40 \\
20m time (s) & $3.13 \pm .09$ & $3.16 \pm .10$ & 0.005 & 0.32 \\
Agility (s) & $9.01 \pm .45$ & $9.12 \pm .46$ & 0.034 & 0.26 \\
Predicted $\mathrm{VO}_{2 \max }\left(\mathrm{ml} \mathrm{kg}^{-1} \mathrm{~min}^{-1}\right)$ & $57.3 \pm 3.5$ & $56.5 \pm 3.2$ & 0.047 & 0.24 \\
Reach flexibility (cm) & $8.8 \pm 7.5$ & $7.1 \pm 7.3$ & 0.039 & 0.23 \\
Traditional flexibility $(\mathrm{cm})$ & $34.2 \pm 8.3$ & $32.5 \pm 8.3$ & 0.059 & 0.20 \\
\hline
\end{tabular}

Selected were named as the 18 players to start on the field. Non-selected players were available for selection. Effect sizes: trivial $<0.2$, small $0.2-0.5$, medium $0.5-0.8$, large $>0.8$.

Table 2 Statistically significant $(p<0.05)$ differences between high and low possession groups $(m e a n \pm$ S.D.)

\begin{tabular}{lcrrr}
\hline & High $(n=36-42)$ & Low $(n=54-59)$ & $p$-Value & Effect size \\
\hline Anthropometric & & & & \\
$\quad$ Height $(\mathrm{cm})$ & $180.1 \pm 6.4$ & $185.3 \pm 7.0$ & 0.001 & 0.78 \\
$\quad$ Mass $(\mathrm{kg})$ & $76.3 \pm 8.4$ & $81.1 \pm 8.9$ & 0.007 & 0.55 \\
$\quad$ Arm length $(\mathrm{cm})$ & $78.4 \pm 4.2$ & $81.0 \pm 5.1$ & 0.008 & 0.56 \\
$\quad$ Standing reach $(\mathrm{cm})$ & $232.9 \pm 9.6$ & $240.7 \pm 101$ & 0.001 & 0.79 \\
Fitness & & & & \\
5 m time $(\mathrm{s})$ & $1.11 \pm .04$ & $1.14 \pm .06$ & 0.024 & 0.56 \\
20 & $3.11 \pm .08$ & $3.15 \pm .10$ & 0.009 & 0.44 \\
Predicted $\dot{V} \mathrm{O}_{2 \max }\left(\mathrm{ml} \mathrm{kg}^{-1} \mathrm{~min}^{-1}\right)$ & $58.8 \pm 3.6$ & $57.0 \pm 3.4$ & 0.031 & 0.51 \\
\hline
\end{tabular}

Effect sizes: trivial $<0.2$, small $0.2-0.5$, medium $0.5-0.8$, large $>0.8$.

abilities, e.g. elite versus sub-elite soccer players ${ }^{9}$ or senior versus junior soccer players. ${ }^{10}$ It would be expected to be more difficult to distinguish between players that are more homogeneous with respect to playing ability. The subjects in this study were the best ARF players for their age in their region, and this is one of the cohorts that the professional AFL clubs draw from. Some of the differ- ences reported here are especially remarkable in $\quad 410$ that the participant group played all of the first ${ }_{411}$ eight games of the season.

\section{Indicators of player performance}

There were significant differences in anthropometric and fitness characteristics between the players

Table 3 Statistically significant differences $(p<0.05)$ between groups based on number of games out of the first eight games earning votes $(1-6)($ mean \pm S.D.)

\begin{tabular}{llll}
\hline & High votes $(n=38-41)$ & Medium votes $(n=31-36)$ & Low votes $(n=21-24)$ \\
\hline $\begin{array}{l}\text { Anthropometric } \\
\text { Arm length }(\mathrm{cm})\end{array}$ & $78.8 \pm 4.2^{\mathrm{a}}(p=0.049)$ & $81.4 \pm 5.4$ & $79.6 \pm 4.9$ \\
Fitness & & & \\
$5 \mathrm{~m}$ time $(\mathrm{s})$ & $1.12 \pm .04^{\mathrm{b}}(p=0.043)$ & $1.12 \pm .07^{\mathrm{b}}(p=0.014)$ & $1.16 \pm .05$ \\
$20 \mathrm{~m}$ time (s) & $3.12 \pm .08^{\mathrm{b}}(p=0.045)$ & $3.12 \pm .10^{\mathrm{b}}(p=0.032)$ & $3.18 \pm .09$ \\
\hline & & \\
a Compared to medium. & &
\end{tabular}


Relationship between pre-season anthropometric and fitness measures and indicators of playing performance in elite junior

Table 4 Statistically significant differences $(p<0.05)$ between top and bottom four teams on the competition ladder (mean \pm S.D.)

\begin{tabular}{lrccc}
\hline & Top $(n=42)$ & Bottom $(n=24)$ & $p$-Value & Effect size \\
\hline $\begin{array}{l}\text { Anthropometric } \\
\quad \text { Mass }(\mathrm{kg})\end{array}$ & $81.8 \pm 9.3$ & $76.7 \pm 9.1$ & 0.032 & 0.55 \\
$\quad$ Standing reach $(\mathrm{cm})$ & $240.1 \pm 9.3$ & $234.3 \pm 12.4$ & 0.038 & 0.53 \\
$\quad$ & & & \\
Fitness & $59.7 \pm 4.6$ & $63.6 \pm 6.5$ & 0.007 & 0.69 \\
$\quad$ VJ $(\mathrm{cm})$ & & & \\
Game statistics & $14.2 \pm 4.3$ & $13.8 \pm 4.9$ & 0.726 & 0.09 \\
$\quad$ Average possessions/game & $3.6 \pm 1.6$ & $3.2 \pm 1.3$ & 0.165 & 0.27 \\
$\quad$ Average marks/game & $3.4 \pm 5.2$ & $4.5 \pm 8.3$ & 0.657 & 0.16 \\
$\quad$ Average hitouts/game & & & \\
\hline
\end{tabular}

Effect sizes: trivial $<0.2$, small $0.2-0.5$, medium $0.5-0.8$, large $>0.8$.

that were selected and not selected to play in the first game of the season (Table 1). The selected players had significantly greater mass, hand span, VJ, predicted $\dot{V}_{2 \text { max }}$, reach flexibility and were faster in $5 \mathrm{~m}$ time, $20 \mathrm{~m}$ time and the agility test. However since all of the effect sizes were between 0.23 and 0.44 , these differences were relatively small in magnitude. Interestingly, the selected players were only $1 \mathrm{~cm}$ taller $(0.5 \%)$ but $3.6 \mathrm{~kg}(4.7 \%)$ heavier than the non-selected group. If this extra mass is primarily muscle tissue, this difference might be a reflection of a greater physical maturity of the selected group. This idea is consistent with the finding of $\mathrm{Keogh}^{4}$ that selected juniors possessed greater mass despite being of a similar age to non-selected players. The relatively small differences between selected and non-selected players in the present study might have been influenced by the method by which players were selected. Although team selection is generally based on perception of playing ability, some clubs indicated that older rather than the best players were given priority in selection for the first game.

The players that acquired the greater number of kicks and handballs (possessions) in the first eight games were significantly shorter, had less body mass, smaller arm length and standing reach. These players were also significantly faster $(5$ and $20 \mathrm{~m}$ time) and had better predicted $\dot{V}_{2 \max }$ (Table 2). This suggests that being smaller and having greater pre-season levels of speed and endurance are an advantage for gaining possessions, whereas greater levels of agility (as measured by the AFL agility test), vertical jump and sit and reach flexibility are not. The results may also be a reflection of the playing position where the smaller individuals have more opportunities to gain possession of the ball. Recent reports of senior AFL players showed that midfielders covered the greatest distances in a game ${ }^{1}$ tended to have better endurance and had lower skinfold measurements than forwards. ${ }^{2}$

As with speed, it might be expected that greater agility would be advantageous for performance indicators such as gaining possessions. However the higher possession group was not significantly better in this component of fitness $(p=0.114)$. This might be explained by a lack of specificity of the agility test used. Game analysis of movement patterns of AFL players ${ }^{1}$ indicated that $80 \%$ or more of the changes of direction involved less than $90^{\circ}$ changes. However the AFL agility test used in the present study required five changes of direction that were all over $90^{\circ}$. Further, recent research on netballers ${ }^{11}$ and on ARF players ${ }^{12}$ has demonstrated that agility tests that contain a need to react to an opponent's movements are more related to performance than tests involving pre-planned movements. The agility test used in this study simply involved running around stationary poles with no reactive component.

The only significant difference in anthropometric or fitness measures between the high and low marking groups was body mass, with the high group being on average $4.5 \mathrm{~kg}(6 \%)$ heavier. This might be explained by a heavier player being able to hold a position in a marking contest. It might be expected that taller players with longer arms, larger hands and better VJ ability would also have an advantage in marking, but this was not indicated by the results. Possibly height is advantageous for contested marks but many marks may be taken in uncontested situations.

Players who achieved at least one hitout per game on average were divided into high and low average hitout groups. The only variable to be significantly different for the two groups was height, with the high hitout group being $5.7 \mathrm{~cm}$ taller on average. The correlation of $r=0.70(p<0.01)$ sup- 
ports the close association between height and the number of hitouts. This provides strong evidence for the importance of height in ruck contests. The lower hitout group was very similar in arm length and VJ ability, which suggests that these variables are not as important as height. Vertical jumping ability from a standing position was not significantly related to either the number of marks or hitouts. While one study indicated the VJ was superior in better players, ${ }^{4}$ most research on ARF has pointed to no significant advantage for performance from $\mathrm{VJ}$ ability when tested from a standing position. ${ }^{2,3,5} \mathrm{It}$ is possible that the ability to jump from a run-up is more related to football performance. ${ }^{2,5}$

Players who played all of the first eight games were divided into three groups on the basis of the number of games where votes were awarded. This comparison revealed that the higher vote winners were significantly faster over 5 and $20 \mathrm{~m}$ sprints, suggesting that acceleration is important for gaining votes. It was also noteworthy that the high vote winners achieved significantly more $(p<0.05)$ average possessions $(17.2 \pm 4.7)$ than the medium group $(12.9 \pm 3.1)$ and the low group $(10.7 \pm 3.5)$. These results imply that players with greater speed and endurance acquire more possessions (as mentioned earlier) and appeal to those that award votes based on performance.

The position of each team on the ladder is a basic indication of team success at a particular point in time. After eight games, the top and bottom four teams were compared. The players from the top four teams tended to be taller, had a greater standing reach and body mass. This provides some evidence for the importance of these anthropometric variables for team success. However the better performing teams did not have players with better pre-season fitness levels (Table 4). In fact, the players from the top four teams were significantly worse in VJ $(6 \%)$. Further, there was no difference between the top and bottom teams in average number of possessions, marks or hitouts (Table 4). This suggests that higher levels of pre-season fitness do not guarantee team success and these game statistical measures are not directly related to team standings, at least after the first eight games.

Both measures of sit and reach flexibility and the AFL agility test showed little relationship with player performance indicators or team standings. The sit and reach test is widely used but has been criticized because it is a general measure of bilateral hamstring flexibility as well as trunk mobility. ${ }^{13}$ Further this test does not seem to be useful for predicting hamstring strains in footballers. ${ }^{14}$ A potentially better test that has been used in ARF is the
$90 / 90^{2}$ because this flexibility test isolates the hamstrings muscle group of each individual leg.

\section{Conclusions}

This study provides evidence that in $\mathrm{U}-18$ footballers, taller players with a greater reach and more body mass have an advantage for certain performance indicators which may contribute to team success. Although selected players had a greater hand span, this variable had a low nonsignificant relationship to other performance indicators (including the number of marks) or team success. Smaller players were associated with the average number of possessions and winning votes.

Overall this analysis suggests that pre-season fitness levels have a significant influence on individual player performance indicators, but not on team performance, at least as measured by the tests in this study. It should be acknowledged that other tests considered to be better measures of various fitness components could reveal different relationships to performance. Players with higher levels of certain fitness components may have an advantage in acquiring possessions, but team success depends on many other factors such as how the ball is used in a game (skill execution), tactical considerations and the opposition. The impact of fitness measures on playing performance needs to be determined by training studies. For example, it would be beneficial to know how various speed, agility and endurance training methods influence playing performance as measured by various game statistical indicators.

\section{Practical implications}

- Improving endurance and the ability to accelerate over $20 \mathrm{~m}$ during the pre-season may be an advantage for gaining possessions and winning votes during the early part of the season.

- Although height may be an advantage for ruck players and weight for marking, the number of hitouts, marks and possessions do not necessarily relate to team success.

- The measurement of hand span, arm length, vertical jump from a standing position, agility as assessed by the Australian Football League agility test, and sit and reach flexibility does not provide a good prediction of individual player performance indicators or team standings after eight games. Therefore, the use of these tests should be questioned for elite under-18 Australian Rules football players.
553 554 555 556 550

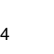

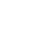
6

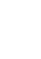

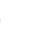
. .

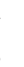
.

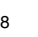
. . .

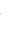
. 
Relationship between pre-season anthropometric and fitness measures and indicators of playing performance in elite junior

\section{Acknowledgement}

The authors wish to thank David Code from Football Victoria for his assistance.

\section{References}

1. Dawson B, Hopkinson R, Appleby B, Stewart G, Roberts C. Player movement patterns and game activities in the Australian Football League. J Sci Med Sport 2004;7(3):278-91.

2. Young WB, Newton RU, Doyle TLA, Chapman D, Cormack S, Stewart G, et al. Physiological and anthropometric characteristics of starters and non-starters and playing positions in elite Australian Rules football: a case study. J Sci Med Sport 2005;8(3):333-45.

3. Marchant DB, Austin R. A comparison of selected fitness parameters of elite under age and elite open age Australian rules footballers. In: Australian conference of science and medicine in sport. 1996. p. 418-9.

4. Keogh J. The use of physical fitness scores and anthropometric data to predict selection in an elite under 18 Australian Rules football team. J Sci Med Sport 1999;2(2):125-33.

5. Pyne DB, Gardner AS, Sheehan K, Hopkins WG. Fitness testing and career progression in AFL football. J Sci Med Sport 2005;8(3):321-32.
6. Ellis L, Gastin P, Lawrence S, Savage B, Buckeridge A, Stapff $A$, et al. Protocols for the physiological assessment of team sport players, Chapter 9. In: Gore CJ, editor. Physiological tests for elite athletes. Champaign: Human Kinetics; 2000.

7. Ramsbottom R, Brewer J, Williams C. A progressive shuttle run test to estimate maximal oxygen uptake. $\mathrm{Br} J$ Sports Med 1988;22:141-5.

8. Cohen J. Statistical power analysis for the behavioural sciences. Hillsdale: L. Eribaum Associates; 1988.

9. Edwards AM, MacFadyen AM, Clark N. Test performance indicators from a single soccer specific fitness test differentiate between highly trained and recreationally active soccer players. J Sports Med Phys Fit 2003;43(1):14-20.

10. Reilly T, Williams AM, Nevill A, Franks A. A multidisciplinary approach to talent identification in soccer. J Sport Sci 2000;18:695-702.

11. Farrow D, Young W, Bruce L. The development of a test of reactive agility for netball: a new methodology. J Sci Med Sport 2005;8(1):52-60.

12. Sheppard JM, Young WB, Doyle TA, Sheppard TA, Newton RU. An evaluation of a new test of reactive agility, and its relationship to sprint speed and change of direction speed. J Sci Med Sport, in press.

13. Kendall FP, McCreary EK, Provance PG. Muscles testing and function. Baltimore: Williams and Wilkins; 1993.

14. Orchard J, Marsden J, Lord S, Garlick D. Preseason hamstring muscle weakness associated with hamstring muscle injury in Australian footballers. Amer J Sports Med 1997;25(1):81-5. 